\title{
Measuring Incompatible Observables by Exploiting Sequential Weak Values
}

\author{
F. Piacentini, A. Avella, M. P. Levi, M. Gramegna, G. Brida, and I. P. Degiovanni \\ INRIM, Strada delle Cacce 91, I-10135 Torino, Italy \\ E. Cohen \\ Wills Physics Laboratory, University of Bristol, Tyndall Avenue, Bristol BS8 1TL, United Kingdom \\ R. Lussana, F. Villa, A. Tosi, and F. Zappa \\ Politecnico di Milano, Dipartimento di Elettronica, Informazione e Bioingegneria, \\ Piazza Leonardo da Vinci 32, 20133 Milano, Italy \\ M. Genovese \\ INRIM, Strada delle Cacce 91, I-10135 Torino, Italy and INFN, Via P. Giuria 1, I-10125 Torino, Italy
}

(Received 4 May 2016; published 20 October 2016)

\begin{abstract}
One of the most intriguing aspects of quantum mechanics is the impossibility of measuring at the same time observables corresponding to noncommuting operators, because of quantum uncertainty. This impossibility can be partially relaxed when considering joint or sequential weak value evaluation. Indeed, weak value measurements have been a real breakthrough in the quantum measurement framework that is of the utmost interest from both a fundamental and an applicative point of view. In this Letter, we show how we realized for the first time a sequential weak value evaluation of two incompatible observables using a genuine single-photon experiment. These (sometimes anomalous) sequential weak values revealed the single-operator weak values, as well as the local correlation between them.
\end{abstract}

DOI: 10.1103/PhysRevLett.117.170402

Measurements are the very basis of physics. In quantum mechanics they assume even a more fundamental role, since observables can have undetermined values that "collapse" on a specific one only when a strong measurement (described by a projection operator) is performed. Furthermore, a crucial feature of quantum measurement is that measuring one observable completely erases the information on its conjugate one (e.g., measurement of position erases information about momentum). This impossibility can be partially relaxed when considering joint or sequential weak value evaluation [1-5]. Weak values, introduced in Ref. [1] and first realized in Refs. [6-8], represent a new quantum measurement paradigm, where only a small amount of information is extracted from a single measurement, so that the state basically does not collapse. They can have anomalous values (imaginary, unbounded values) and, while their real part is usually interpreted as a conditional average of the observable in the limit of zero disturbance [9], their imaginary part is related to the disturbance (or backaction) of the measuring pointer during the measurement process [10]. Weak values have been used for addressing fundamental questions [11] such as contextuality [12,13], but can also be seen as a ground-breaking tool for quantum metrology allowing high-precision measurements (at least in presence of specific noises [14]), as the tiny spin Hall effect [8] or small beam deflections [15] and characterization of wave function [16-18].

Nevertheless, up to now only weak measurements (WMs) on a single observable (eventually followed by a strong measurement) or joint WMs performed on commuting observables and on different particles (or optical modes) have been realized experimentally [6-8,11,12,14-27]. However, sequential weak values, which are more sensitive to the system's dynamics and whose time order is crucial, have not been performed yet. One of the most intriguing properties of sequential weak values is that they allow the simultaneous measurement of noncommuting observables [4] over an ensemble of single photons. This result has not been reached in any previous experiment, since none of them allowed simultaneous (weak) measurement of noncommuting observables [28]. Here we achieve this result by experimentally demonstrating the peculiar predictions regarding single and sequential weak values, measuring at the same time noncompatible polarizations using real single photons.

Specifically, the weak value of an observable $\hat{A}$ is defined as $\langle\hat{A}\rangle_{w}=\frac{\left\langle\psi_{f}|\hat{A}| \psi_{i}\right\rangle}{\left\langle\psi_{f} \mid \psi_{i}\right\rangle}$, where a key role is symmetrically played by the preselected $\left(\left|\psi_{i}\right\rangle\right)$ and postselected $\left(\left|\psi_{f}\right\rangle\right)$ quantum states. When the pre- and postselected states are equal, the weak value is just the expectation value of $\hat{A}$.

Weak values are usually obtained taking advantage of the coupling between the observable $\hat{A}$ and the pointer observable $\hat{P}$, according to the unitary transformation $\hat{U}=\exp (-i g \hat{A} \otimes \hat{P})$. When the weak interaction regime is assumed, one can describe the evolution of this system, prepared in the preselected state and projected on the postselected state, as 


$$
\left\langle\psi_{f}\left|e^{-i g \hat{A} \otimes \hat{P}}\right| \psi_{i}\right\rangle \simeq\left\langle\psi_{f} \mid \psi_{i}\right\rangle\left(\mathbf{1}-i g\langle\hat{A}\rangle_{w} \hat{P}\right) .
$$

By measuring the observable $\hat{X}$ - canonically conjugated to $\hat{P}$-one can extract, in general, the real part of the weak value $\langle\hat{A}\rangle_{w}$ from the relation $\langle\hat{X}\rangle=\operatorname{Re}\left[g\langle\hat{A}\rangle_{w}\right]$ (and the weak value itself if $\operatorname{Re}\left[\langle\hat{A}\rangle_{w}\right]=\langle\hat{A}\rangle_{w}$ ), given that $g$ is independently estimated.

Measurements of joint [3] or sequential [4] weak values of two observables $\hat{A}$ and $\hat{B}$ are obtained when two different couplings ( $g_{x}$ and $g_{y}$ ) to two distinct pointer observables (in our experiment the two transverse momenta $\hat{P}_{x}$ and $\hat{P}_{y}$ ) are realized between the pre- and postselection of the state. In particular, if the measurement is performed exploiting simultaneous interactions, we are dealing with measurement of the joint weak value, and by measuring the covariance of the position observables $\hat{X}$ and $\hat{Y}(\langle\hat{X} \hat{Y}\rangle)$ one obtains [3]

$$
\langle\hat{X} \hat{Y}\rangle=\frac{1}{4} g_{x} g_{y} \operatorname{Re}\left[\langle\hat{A} \hat{B}+\hat{B} \hat{A}\rangle_{w}+2\langle\hat{A}\rangle_{w}^{*}\langle\hat{B}\rangle_{w}\right],
$$

while if we have a sequence of two weak interactions, e.g., the first interaction is described by the unitary transformation $\hat{U}_{x}=\exp \left(-i g_{x} \hat{A} \otimes \hat{P}_{x}\right)$ and the second by $\hat{U}_{y}=\exp \left(-i g_{y} \hat{B} \otimes \hat{P}_{y}\right)$, when measuring $\langle\hat{X} \hat{Y}\rangle$ one obtains [4]

$$
\langle\hat{X} \hat{Y}\rangle=\frac{1}{2} g_{x} g_{y} \operatorname{Re}\left[\langle\hat{A} \hat{B}\rangle_{w}+\langle\hat{A}\rangle_{w}^{*}\langle\hat{B}\rangle_{w}\right] .
$$

We can already see that the procedure for estimating the sequential weak value $\langle\hat{A} \hat{B}\rangle_{w}$ is strictly different from the usual procedure for estimating the single weak value of the product operator $\hat{A} \hat{B}$, which corresponds to a single displacement of some measuring pointer. Here, the result is proportional to the correlation between two pointers' displacements $\hat{X}$ and $\hat{Y}$. It thus corresponds to the weak values of the operators $\hat{A}$ and $\hat{B}$, as well as the temporal correlation between them. In addition, when $\hat{A}$ and $\hat{B}$ are noncommuting, the product $\hat{A} \hat{B}$ is non-Hermitian; hence, the weak coupling to it leads to a nonunitary evolution in time, while in our approach the two separate weak couplings to $\hat{A}$ and $\hat{B}$ lead to unitary evolution in time. Intriguing schemes exploiting sequential weak averages for the direct measurement of density functions are discussed in Ref. [5] (where, indeed, it is shown that sequential weak values are necessary, specifically, in that case, for measuring the weak average obtained from a sequence of two weak interactions plus a strong measurement).

Thus, the real part of sequential $\left(\operatorname{Re}\left[\langle\hat{A} \hat{B}\rangle_{w}\right]\right)$ or joint $\left(\operatorname{Re}\left[\langle\hat{A} \hat{B}+\hat{B} \hat{A}\rangle_{w}\right]\right)$ weak values can be evaluated by measuring $\langle\hat{X} \hat{Y}\rangle$ and by evaluating each weak value independently, i.e., $\langle\hat{A}\rangle_{w}$ and $\langle\hat{B}\rangle_{w}$ (these can be obtained by measuring the mean values of the positions and momenta $\langle\hat{X}\rangle,\langle\hat{Y}\rangle,\left\langle\hat{P}_{x}\right\rangle$, and $\left.\left\langle\hat{P}_{y}\right\rangle[3,4]\right)$.
In our experiment, we focus on the case of sequential weak values measurement, where the operators $\hat{A}$ and $\hat{B}$ are the linear projectors $\hat{\Pi}_{V}=|V\rangle\langle V|$ and $\hat{\Pi}_{\psi}=|\psi\rangle\langle\psi|$ (with $|\psi\rangle=\cos \theta|H\rangle+\sin \theta|V\rangle$ ). The considered quantum system is a (heralded) single photon prepared (preselected) in the initial state $\left.\left|\phi_{i}\right\rangle\right\rangle=\left|\psi_{i}\right\rangle \otimes\left|f_{x}\right\rangle \otimes\left|f_{y}\right\rangle$, with $\left|\psi_{i}\right\rangle=\cos \theta_{i}|H\rangle+\sin \theta_{i}|V\rangle$ and $\left|f_{\xi}\right\rangle=\int \mathrm{d} \zeta \mathcal{F}_{\xi}(\zeta)|\zeta\rangle$, where $\left|\mathcal{F}_{\xi}(\zeta)\right|^{2}$ is the probability density function of detecting the photon in the position $\xi$ (with $\xi=x, y$ ) of the transverse spatial plane. $\left|\mathcal{F}_{\xi}(\zeta)\right|^{2}$ in our experiment is reasonably Gaussian, since the single photon guided in a single-mode optical fiber is collimated with a telescopic optical system. By experimental evidence, we can assume that the (unperturbed) $\left|\mathcal{F}_{\xi}(\zeta)\right|^{2}$ is centered around zero and has the same width $\sigma$ both for $\xi=x$ and for $\xi=y$.

The single photons undergo two sequential weak interactions inducing displacements in two orthogonal directions according to the unitary transformations $\hat{U}_{y}=\exp \left(-i g_{y} \hat{\Pi}_{V} \otimes \hat{P}_{y}\right)$ and $\hat{U}_{x}=\exp \left(-i g_{x} \hat{\Pi}_{\psi} \otimes \hat{P}_{x}\right)$. This spatial displacement-due to the polarization-sensitive spatial walk-off of the Poynting vector of the single photon induced by its propagation into a birefringent mediumrealizes in practice the weak interaction (see Fig. 1).

Then, the single photon is projected on the postselected linear polarization state $\left|\psi_{f}\right\rangle$ and detected by a spatialresolving detector. Thus, the post-selected single-photon state is $\left.\left.\left|\phi_{f}\right\rangle\right\rangle=\left\langle\psi_{f}\left|\hat{U}_{x} \hat{U}_{y}\right| \psi_{i}\right\rangle\right\rangle$. Since we are focusing on linear polarizations only, it is possible to evaluate the sequential weak value of the (in general) noncommuting projectors $\left\langle\hat{\Pi}_{\psi} \hat{\Pi}_{V}\right\rangle_{w}$, as well as the single weak values $\left\langle\hat{\Pi}_{\psi}\right\rangle_{w}$ and $\left\langle\hat{\Pi}_{V}\right\rangle_{w}$. In fact, according to Eq. (3), we have $\langle\hat{X} \hat{Y}\rangle=\frac{1}{2} g_{x} g_{y}\left(\left\langle\hat{\Pi}_{\psi} \hat{\Pi}_{V}\right\rangle_{w}+\left\langle\hat{\Pi}_{\psi}\right\rangle_{w}\left\langle\hat{\Pi}_{V}\right\rangle_{w}\right),\langle\hat{X}\rangle=g_{x}\left\langle\hat{\Pi}_{\psi}\right\rangle_{w}$, $\langle\hat{Y}\rangle=g_{y}\left\langle\hat{\Pi}_{V}\right\rangle_{w}$ [29]. By inverting these relations, it is possible to obtain the weak values of the two noncommuting observables $\left\langle\hat{\Pi}_{V}\right\rangle_{w}$ and $\left\langle\hat{\Pi}_{\psi}\right\rangle_{w}$, as well as the sequential weak value of the two noncommuting observables $\left\langle\hat{\Pi}_{\psi} \hat{\Pi}_{V}\right\rangle_{w}$. Note that this relation between position mean values and polarization weak values holds only in the case of weak interaction, i.e., only for $g / \sigma \ll 1\left(g_{x} / \sigma \sim g_{y} / \sigma \sim 0.15\right.$ in our case).

The experimental setup is presented in Fig. 1: it hosts a heralded single-photon source based on pulsed parametric down-conversion (PDC), exploiting a $796 \mathrm{~nm}$ mode-locked Ti:Sapphire laser (repetition rate: $76 \mathrm{MHz}$ ) whose second

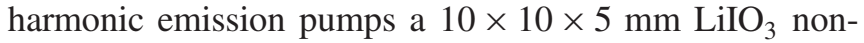
linear crystal, producing Type-I PDC.

The idler photon $\left(\lambda_{i}=920 \mathrm{~nm}\right)$ is coupled to a single-mode fiber (SMF) and then addressed to a silicon single-photon avalanche detector (SPAD), heralding the presence of the correlated signal photon $\left(\lambda_{s}=702 \mathrm{~nm}\right)$ that, after being SMF coupled, is sent to a launcher and then to the free-space optical path, where the experiment for weak values evaluation is performed (see Ref. [30] for details). 


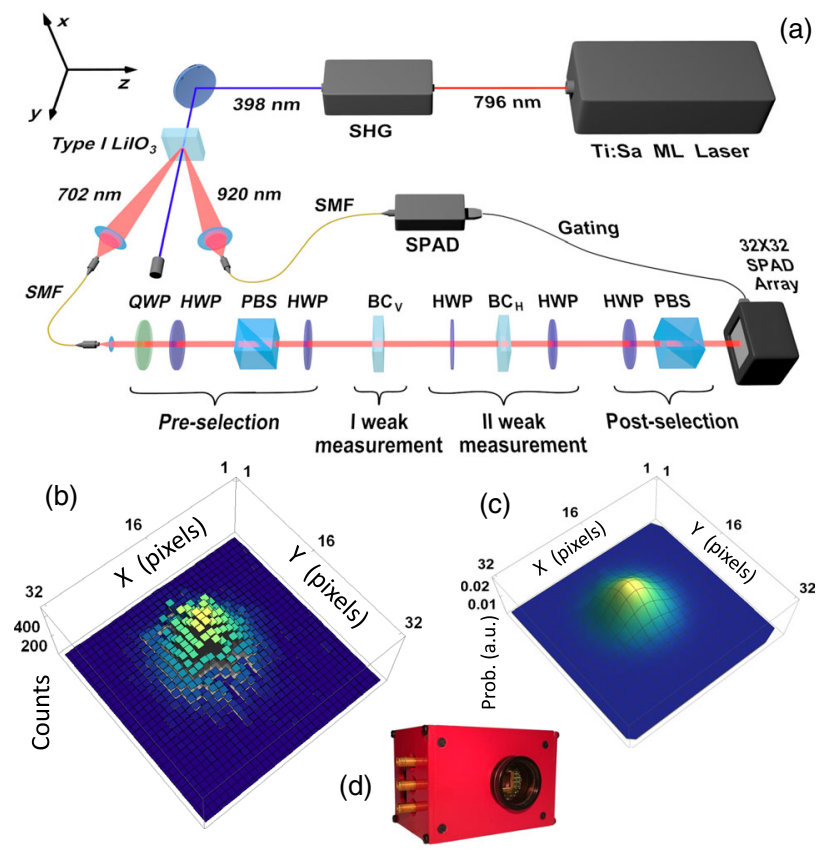

FIG. 1. (a) Experimental setup and detection apparatus: second harmonic generator (SHG), quarter wave plate (QWP), half wave plate (HWP), polarizing beam splitter (PBS), birefringent crystal (BC). (b) Typical single data acquisition obtained with our spatial resolving detector $(32 \times 32$ SPAD camera), after noise subtraction (acquisition time: $300 \mathrm{~s}$ ). (c) The corresponding, theoretically predicted probability distribution. (d) Our SPAD camera prototype.

After the launcher, the heralded single photon state is collimated by a telescopic system, and then prepared (preselected) in a linear polarization state $\left|\psi_{i}\right\rangle$ (by means of a calcite polarizer followed by a half-wave plate). The first weak interaction is carried out by a 2-mm long birefringent crystal $\left(\mathrm{BC}_{V}\right)$, whose extraordinary $(e)$ optical axis lies in the $Y-Z$ plane with an angle of $\pi / 4$ with respect to the $Z$ direction. Because of the spatial walk-off effect experienced by the vertically polarized photons (i.e., along the $Y$ direction), horizontal- and vertical-polarization paths get slightly separated along the $Y$ direction, inducing in the initial state $\left|\psi_{i}\right\rangle$ a small decoherence [30], leaving it substantially unaffected.

Together with the spatial walk-off, the birefringent crystal also induces on this single-photon state a temporal walk-off and eventually a polarization change, both to be eliminated in order to avoid unwanted additional decoherence effects (details in Ref. [30]).

After this, the photon goes to the second weak interaction module. It is constituted by a system $\left(\mathrm{BC}_{H}\right)$ of two birefringent crystals rotated by $90^{\circ}$ with respect to the previous one; i.e., the first crystal has its optical axis in the $X-Z$ plane, while the second one has the optical axis in the $Y$ direction, inserted between two half-wave plates. By rotating both wave plates of the same angle with respect to the $H$ axis, one obtains the weak interaction on the linear polarization state $|\psi\rangle$ with the polarizations separation appearing along the $X$ direction. This can be thought of as a simple example of the unitary evolution between weak interactions affecting the sequential weak value, as discussed in Ref. [4].

After both WMs are performed, the photon meets a halfwave plate and a calcite polarizer, projecting the state onto the postselected state $\left|\psi_{f}\right\rangle$, and then it is detected by a spatial-resolving single-photon detector prototype. This device is a two-dimensional array made of $32 \times 32$ "smart pixels"-each pixel includes a SPAD and its front-end electronics for counting and timing single photons [30,32].

The main results of our work are summarized in Fig. 2, where we have chosen [based on the standard weak value formula, as well as Eq. (3)] the pre- and postselected states in order to show paradoxical properties predicted for sequential weak values, namely, $\left|\psi_{i}\right\rangle=0.588|H\rangle+$ $0.809|V\rangle$ and $\left|\psi_{f}\right\rangle=|H\rangle$ in Fig. 2(a), and $\left|\psi_{i}\right\rangle=$ $0.509|H\rangle+0.861|V\rangle$ and $\left|\psi_{f}\right\rangle=-0.397|H\rangle+0.918|V\rangle$ in Fig. 2(b). Here we plot the two weak values and the sequential one as a function of the angle $\theta$ of the polarization projector $\hat{\Pi}_{\psi}$ of the second weak interaction, showing a remarkable agreement with the theoretical predictions. An example of a paradoxical situation is represented by the case where, even if one of the two single weak values is zero (within the uncertainty), the sequential weak value of the two noncommuting observables is significantly different from zero, e.g., in Fig. 2(a) when $\theta=0.2 \pi$ we obtain $\left\langle\hat{\Pi}_{V}\right\rangle_{w}=0.03 \pm 0.03$, $\left\langle\hat{\Pi}_{\psi}\right\rangle_{w}=1.44 \pm 0.04$, while $\left\langle\hat{\Pi}_{\psi} \hat{\Pi}_{V}\right\rangle_{w}=0.69 \pm 0.15$, or when $\theta=0.9 \pi$ we have $\left\langle\hat{\Pi}_{V}\right\rangle_{w}=0.04 \pm 0.03,\left\langle\hat{\Pi}_{\psi}\right\rangle_{w}=$ $0.35 \pm 0.04$, while $\left\langle\hat{\Pi}_{\psi} \hat{\Pi}_{V}\right\rangle_{w}=-0.46 \pm 0.10$. In particular, in the last case, we have a positive and an almost null positive single weak value associated to the two noncommuting observables, while the corresponding
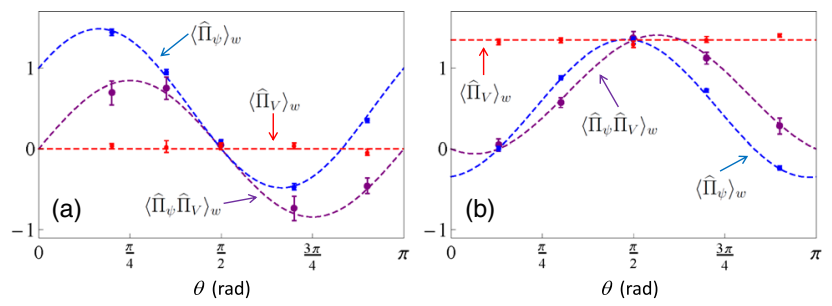

FIG. 2. Measured weak values (data points) compared with the theoretical predictions (dashed lines) for different $\hat{\Pi}_{\psi}$ (i.e., for different values of $\theta$, since $|\psi\rangle=\cos \theta|H\rangle+\sin \theta|V\rangle)$. Blue and red points and lines correspond to the evaluations of the single weak values $\left\langle\hat{\Pi}_{\psi}\right\rangle_{w}$ and $\left\langle\hat{\Pi}_{V}\right\rangle_{w}$, respectively, while purple points and line represent the evaluation of the sequential weak value $\left\langle\hat{\Pi}_{\psi} \hat{\Pi}_{V}\right\rangle_{w}$. Uncertainty bars are evaluated on the basis of sequences of repeated measurements. The pre-selected and post-selected states are, respectively, $\left|\psi_{i}\right\rangle=0.588|H\rangle+0.809|V\rangle$ and $\left|\psi_{f}\right\rangle=|H\rangle$ for plot (a), and $\left|\psi_{i}\right\rangle=0.509|H\rangle+0.861|V\rangle$ and $\left|\psi_{f}\right\rangle=-0.397|H\rangle+0.918|V\rangle$ for plot (b). 
sequential weak value is negative, and with a modulus 2 orders of magnitude greater than the product of the single weak values. We also observe the surprising situation of having one of the single weak values and the sequential weak value both positive, while the other single weak value is negative [e.g., in Fig. 2(b) when $\theta=0.9 \pi$ we obtain $\left\langle\hat{\Pi}_{V}\right\rangle_{w}=1.40 \pm 0.04, \quad\left\langle\hat{\Pi}_{\psi} \hat{\Pi}_{V}\right\rangle_{w}=0.28 \pm 0.10$, while $\left\langle\hat{\Pi}_{\psi}\right\rangle_{w}=-0.24 \pm 0.03$ ]. Along the lines of Ref. [11], these are clear demonstrations of the "product rule" breakdown when weak values are concerned.

More generally, looking at Fig. 2(a) we can note that, despite the fact that $\left\langle\hat{\Pi}_{V}\right\rangle_{w} \sim 0$ everywhere, we have that both the single weak value of the other noncommuting observable and the sequential one are significantly nonzero. Furthermore, for both of them we have observed anomalous weak values, i.e., weak values not bounded by the observables spectrum (in our case between 0 and 1). In Fig. 2(a) we observe $\left\langle\hat{\Pi}_{\psi}\right\rangle_{w}>1$ and $\left\langle\hat{\Pi}_{\psi}\right\rangle_{w}<0$, as well as $\left\langle\hat{\Pi}_{\psi} \hat{\Pi}_{V}\right\rangle_{w}<0$. Analogously, in Fig. 2(b) we find in one case that all the weak values $\left\langle\hat{\Pi}_{\psi}\right\rangle_{w},\left\langle\hat{\Pi}_{V}\right\rangle_{w}$, and $\left\langle\hat{\Pi}_{\psi} \hat{\Pi}_{V}\right\rangle_{w}$ are larger than 1.

As pointed out also in Ref. [4], weak values present an internal consistency; thus, they should be considered as the actual values of the parameters measured albeit the curious appearance of anomalous values. This internal consistency is also reflected in our data. In Fig. 2(a) looking at the data corresponding to $\theta=0.2 \pi$ (in the following $\hat{\Pi}_{\psi_{0}}$ ) and $\theta=0.7 \pi$ (in the following $\hat{\Pi}_{\psi_{0}^{\perp}}$ ) we observe that $\left\langle\hat{\Pi}_{\psi_{0}}\right\rangle_{w}+\left\langle\hat{\Pi}_{\psi_{0}}^{\perp}\right\rangle_{w}=0.97 \pm 0.06$ in agreement with the general rule $\left\langle\hat{\Pi}_{\psi}\right\rangle_{w}+\left\langle\hat{\Pi}_{\psi}^{\perp}\right\rangle_{w}=1$. Analogously, since generally $\left\langle\hat{\Pi}_{\psi} \hat{\Pi}_{\varphi}\right\rangle_{w}+\left\langle\hat{\Pi}_{\psi}^{\perp} \hat{\Pi}_{\varphi}\right\rangle_{w}=\left\langle\hat{\Pi}_{\varphi}\right\rangle_{w}$, in our case we observe that $\left\langle\hat{\Pi}_{\psi_{0}} \hat{\Pi}_{V}\right\rangle_{w}+\left\langle\hat{\Pi}_{\psi_{0}}^{\perp} \hat{\Pi}_{V}\right\rangle_{w}=-0.05 \pm 0.22$, in agreement with the theoretical prediction $\left(\left\langle\hat{\Pi}_{V}\right\rangle_{w}=0\right)$, and the experimentally measured average value $\left(\left\langle\hat{\Pi}_{V}\right\rangle_{w}=0.02 \pm 0.06\right)$.

Our uncertainties on the weak values presented in the letter and shown in the plots of Fig. 2 are obtained with the uncertainty propagation standard rules (coverage factor $k=1$ ) starting from the images collected by our $32 \times 32$ SPAD array. The statistical fluctuations on our data are obtained collecting 9 different images for each experimental point. After analyzing every image by itself, for each of the quantities $g_{x}, g_{y},\langle\hat{X}\rangle_{f},\langle\hat{Y}\rangle_{f}$, and $\langle\hat{X} \hat{Y}\rangle_{f}$ we extract the mean value and the corresponding uncertainty, i.e., the standard deviation on the average.

Summarizing, we demonstrate an unprecedented measurement capability, providing information on two noncommuting observables at the same time, as well as on the correlation between them, a feature forbidden in the conventional (i.e., POVM-based) measurement framework of quantum mechanics.

In our sequential weak value experiment we exploit two weak couplings plus a "strong" postselection measurement to obtain the simultaneous estimation of two single-operator weak values in connection with the same uncollapsed initial state, as well as the sequential weak value of two (in general, noncommuting) observables. This is more significant (as discussed for instance in Ref. [4] and in the recent Ref. [33]) than what can be obtained from a single weak interaction plus a strong postselection measurement, namely, only a singleoperator weak value estimation and nothing else. Indeed, another weak value means more (noncounterfactual) information and interesting temporal correlations between noncommuting operators, including anomalous and paradoxical weak values.

Furthermore, we note that single-operator weak value estimation exploiting a single weak interaction plus a strong measurement allows obtaining partial information about the complementary observables. For instance, one can employ a weak interaction for the first observable and then perform a strong final measurement on the second, in general complementary, observable. This was essentially the idea behind, e.g., wave function direct characterization experiments [16-18]. Nevertheless, sequential weak values are much richer, allowing one to obtain the single weak values of two (in our case) or more observables, as well as the sequential weak value of, in general, noncommuting observables at the same time, i.e., as a sequence of weak couplings on one and the same photon. This is possible due to the presence of two independent and distinguishable weak interactions before the final strong measurement. Sequential weak values can be used in direct measurement of the density matrix [34], and also in quantum process tomography [35], which makes use of this very technique of estimating an unknown dynamics without considerably changing it.

It is also worth mentioning that our experiment does not only shed light on counterfactual computation [36], but in fact enables for the first time its careful experimental test. As proposed in Ref. [4], the measurement outcome $\left|\psi_{f}\right\rangle$ is counterfactual if it determines the computer's outcome and if the sequential weak value of projections onto all of the "on" instances is zero.

This work has been supported by EMPIR-14IND05 "MIQC2" (the EMPIR initiative is co-funded by the EU H2020 and the EMPIR Participating States) and the EU FP7 under Grant Agreement No. 308803 (project "BRISQ2"). E. C. was supported by ERC AdG NLST and by the Israel Science Foundation Grant No. 1311/14. We wish to thank Yakir Aharonov and Avshalom C. Elitzur for helpful discussions.

Note added.-Recently, Ref. [34] appeared performing an experiment exploiting sequential weak values in an optical setup similar to ours. The authors implemented their sequential weak values experiment performing, as a proof of principle, the direct measurement of the polarization 
density matrix (of a single photon) using also the imaginary part of the weak value, where nevertheless, for simplicity, the single photon source was replaced with a laser beam.

[1] Y. Aharonov, D. Z. Albert, and L. Vaidman, How the Result of a Measurement of a Component of the Spin of a Spin-1/2 Particle Can Turn Out To Be 100, Phys. Rev. Lett. 60, 1351 (1988).

[2] A. G. Kofman, S. Ashhab, and F. Nori, Nonperturbative theory of weak pre-and post-selected measurements, Phys. Rep. 520, 43 (2012).

[3] K. J. Resch and A. M. Steinberg, Extracting Joint Weak Values with Local, Single-Particle Measurements, Phys. Rev. Lett. 92, 130402 (2004).

[4] G. Mitchison, R. Jozsa, and S. Popescu, Sequential weak measurement, Phys. Rev. A 76, 062105 (2007).

[5] J. S. Lundeen and C. Bamber, Procedure for Direct Measurement of General Quantum States Using Weak Measurement, Phys. Rev. Lett. 108070402 (2012).

[6] N. W. M. Ritchie, J. G. Story, and R. G. Hulet, Realization of a Measurement of a “Weak Value," Phys. Rev. Lett. 66, 1107 (1991).

[7] G. J. Pryde, J. L. O'Brien, A. G. White, T. C. Ralph, and H. M. Wiseman, Measurement of Quantum Weak Values of Photon Polarization, Phys. Rev. Lett. 94, 220405 (2005).

[8] O. Hosten and P. Kwiat, Observation of the spin Hall effect of light via weak measurements, Science 319, 787 (2008).

[9] J. Dressel, S. Agarwal, and A. N. Jordan, Contextual Values of Observables in Quantum Measurements, Phys. Rev. Lett. 104, 240401 (2010).

[10] J. Dressel and A. N. Jordan, Significance of the imaginary part of the weak value, Phys. Rev. A 85, 012107 (2012).

[11] Y. Aharonov, A. Botero, S. Popescu, B. Reznik, and J. Tollaksen, Revisiting Hardy's paradox: counterfactual statements, real measurements, entanglement and weak values, Phys. Lett. A 301, 130 (2002).

[12] M. Pusey, Anomalous Weak Values are Proofs of Contextuality, Phys. Rev. Lett. 113, 200401 (2014).

[13] F. Piacentini et al., Experiment Investigating the Connection between Weak Values and Contextuality, Phys. Rev. Lett. 116, 180401 (2016).

[14] J. Dressel, M. Malik, F. M. Miatto, A. N. Jordan, and R. W. Boyd, Colloquium: Understanding quantum weak values: Basics and applications, Rev. Mod. Phys. 86, 307 (2014).

[15] K. J. Resch, Amplifying a tiny optical effect, Science 319, 733 (2008).

[16] J. Z. Salvail, M. Agnew, A. S. Johnson, E. Bolduc, J. Leach, and R. W. Boyd, Full characterization of polarization states of light via direct measurement, Nat. Photonics 7, 316 (2013).

[17] J. S. Lundeen, B. Sutherland, A. Patel, C. Stewart, and C. Bamber, Direct measurement of the quantum wavefunction, Nature (London) 474, 188 (2011).

[18] M. Malik, M. Mirhosseini, M. P. J. Lavery, J. Leach, M. J. Padgett, and R. W. Boyd, Direct measurement of a 27-dimensional orbital-angular-momentum state vector, Nat. Commun. 5, 3115 (2014).
[19] L. A. Rozema, A. Darabi, D. H. Mahler, A. Hayat, Y. Soudagar, and A. M. Steinberg, Violation of Heisenberg's Measurement-Disturbance Relationship by Weak Measurements, Phys. Rev. Lett. 109, 100404 (2012).

[20] R. Mir, J. S. Lundeen, M. W. Mitchell, A. M. Steinberg, J. L. Garretson, and H. M. Wiseman, A double-slit 'which-way' experiment on the complementarity-uncertainty debate, New J. Phys. 9, 287 (2007).

[21] K. Yokota, T. Yamamoto, M. Koashi, and N. Imoto, Direct observation of Hardy's paradox by joint weak measurement with an entangled photon pair, New J. Phys. 11, 033011 (2009).

[22] K. J. Resch, J. S. Lundeen, and A. Steinberg, Experimental realization of the quantum box problem, Phys. Lett. A 324 125 (2004).

[23] A. Danan, D. Farfurnik, S. Bar-Ad, and L. Vaidman, Asking Photons Where They Have Been, Phys. Rev. Lett. 111, 240402 (2013).

[24] M. E. Goggin, M. P. Almeida, M. Barbieri, B. P. Lanyon, J. L. O'Brien, A. G. White, and G. J. Pryde, Violation of the Leggett-Garg inequality with weak measurements of photons, Proc. Natl. Acad. Sci. U.S.A. 108, 1256 (2011).

[25] P. B. Dixon, D. J. Starling, A. N. Jordan, and J. C. Howell, Ultrasensitive Beam Deflection Measurement via Interferometric Weak Value Amplification, Phys. Rev. Lett. 102, 173601 (2009).

[26] J. M. Hogan, J. Hammer, S.-W. Chiow, S. Dickerson, D. M. S. Johnson, T. Kovachy, S. Sugarbaker, and M. A. Kasevich, Precision angle sensor using an optical lever inside a Sagnac interferometer, Opt. Lett. 36, 1698 (2011).

[27] O. S. Magana-Loaiza, M. Mirhosseini, B. Rodenburg, and R. W. Boyd, Amplification of Angular Rotations Using Weak Measurements, Phys. Rev. Lett. 112, 200401 (2014).

[28] Simultaneous measurements of noncommuting observables have been already claimed in the past. For instance, in the Letter [G. A. Howland, J. Schneeloch, D. J. Lum, and J. C. Howell, Simultaneous Measurement of Complementary Observables with Compressive Sensing, Phys. Rev. Lett. 112, 253602 (2014)], the authors present a technique able to perform the direct characterization of the wave function as in Refs. [16-18]. Specifically, Ref. [17] exploits a single weak interaction, as well as a strong final measurement to reconstruct the wave function. The Letter exploits a partial filtering (that cannot be considered "weak"), as well as a strong momentum measurements. The analogy between the two approaches is evident. Here we would like to underline that none of the two approaches is able to provide the estimation of the sequential weak value (of two noncommuting operators), at most they are able to provide the sequential weak average [5].

[29] This enables us to infer the particle's properties via quantum correlations (second order in $g$ ), rather than via the single operator value (first order in $g$, where $g$ is the coupling strength of the von Neumann interaction Hamiltonian). Single weak values usually emerge as first order in $g$, when linearizing the time evolution created by this weak coupling. Sequential weak values are unique in invoking the next order in $g$, which corresponds to (local) correlations between the two measured observables. This resembles joint weak measurements performed on two entangled particles, with the important difference that now the two measurements are performed on 
one and the same particle and thus measure temporal, local correlations where the order of operators is important.

[30] See Supplemental Material at http://link.aps.org/ supplemental/10.1103/PhysRevLett.117.170402, which includes Ref. [31], for details on the experimental setup.

[31] P. Grangier, G. Roger, and A. Aspect, Experimental evidence for a photon anticorrelation effect on a beam splitter: A new light on single-photon interferences, Europhys. Lett. 1, 173 (1986).

[32] F. Villa et al., CMOS imager with 1024 SPADs and TDCs for single-photon timing and 3-D time-of-flight, IEEE J. Sel. Top. Quantum Electron. 20, 364 (2014).
[33] L. Diosi, Structural features of sequential weak measurements, Phys. Rev. A 94, 010103(R) (2016).

[34] G. S. Thekkadath, L. Giner, Y. Chalich, M. J. Horton, J. Banker, and J. S. Lundeen, Direct Measurement of the Density Matrix of a Quantum System, Phys. Rev. Lett. 117, 120401 (2016).

[35] R. Ber, S. Marcovitch, O. Kenneth, and B. Reznik, Process tomography for systems in a thermal state, New J. Phys. 15, 013050 (2013).

[36] G. Mitchison and R. Jozsa, Counterfactual computing, Proc. R. Soc. A 457, 1175 (2001). 